\title{
TUNABLE BANDPASS FILTER USING RF MEMS SWITCHES
}

\author{
A.H.M. ZAHIRUL ALAM*, MD. RAFIQUL ISLAM, SHEROZ KHAN, \\ NiK NoOR ATIKAH NIK MOHD. SALlEH AND NorAINI AZIZ \\ Wireless Communication and Signal Processing Research Group, \\ Electrical and Computer Engineering Department, Faculty of Engineering, \\ International Islamic University Malaysia, \\ Jalan Gombak, 53100 Kuala Lumpur, Malaysia \\ *E-mail : zahirulalam@iium.edu.my
}

\begin{abstract}
A band pass tunable RF filter is proposed by using Radio Frequency (RF) Microelectro Mechanical Systems (MEMS). The tunability is obtained by using capacitive MEMS switches that can be tuned within the bandwidth of $3.6 \mathrm{GHz}$ to $4.4 \mathrm{GHz}$. The performance of the filter depends on geometry and location and types of the MEMS switches. Optimization has been done to achieve tunability by using 3-D high frequency electromagnetic simulator (HFSS).
\end{abstract}

KEYWORDS: Microelectro Mechanical Systems (MEMS), Radio Frequency (RF), Bandpass Filter, Tunable Filter

\section{INTRODUCTION}

Modern wireless/satellite communication, radar, electronic warfare, and instrumentation, all demand tunable filters for flexible and adaptive operations over wide frequency range [1-3]. Mechanically and magnetically tunable band pass filters, although widely employed and handle high power, provide just low to marginal tuning speed, and are bulky due to their macroscale tuning mechanism. Electronically tuned filters, with compactness and quick tuning, are good candidates for highly integrated systems. However, due to losses and nonlinearity associated with p-n junctions and MOS structures and the complexity in biasing, microwave control devices based on these mechanisms may lack of appeal to current wireless communication applications, and thus investigations into tunable filters based on diodes and transistors have barely reported.

Recently, RF MEMS tunable filters of various tuning topologies [4-7], with much lower insertion loss (IL), nonlinearity and higher Q factor than semiconductor based devices, have been demonstrated as an enabling technology for low cost and high performance integrated military or commercial RF systems. These surface micromachined devices have the advantages of simple (electrostatic) tuning with ultra-low tuning power consumption, large bandwidth and capability of monolithic integration with active circuit. Existing designs suffer from passband attenuation mostly due to the architecture itself rather than conductor or dielectric loss. 
Reconfigurable band pass filters (BPFs) draw much attention in modern systems because of their diversity. Reconfigurable BPFs with tunable bandwidth [8, 9], tunable center frequency [10], and switch ability [9] are reported. Among these researches, PIN diodes, piezoelectric transducers, and MEMS cantilever/bridge structures are used as the switch components. In this paper, RF MEMS switches have been proposed to design a reconfigurable interdigital band pass filter and describe the optimization procedure to obtain tunable bandwidth.

\section{DESIGN AND ANALYSIS}

Interdigital bandpass filters are commonly used for microstrip implementation. The filter configuration consists of an array of $n$ Transverse Electro Magnetic (TEM) -mode transmission line resonators, each of which has an electrical length of 90at the midband frequency and is short-circuited at one end and open-circuited at the other with alternative orientation. The coupling is achieved by way of the fields fringing between adjacent resonators separated by spacing [11]. Each line element serves as a resonator, except for the input and output line elements, which have an impedance-matching function . The input and output are also using tapped line which offer space and cost-saving advantages because the first and the last resonators are eliminated. These types of bandpass filters require use of grounding microstrip resonators which can be achieved via holes. The general structure for interdigital filter is shown in Fig. 1.

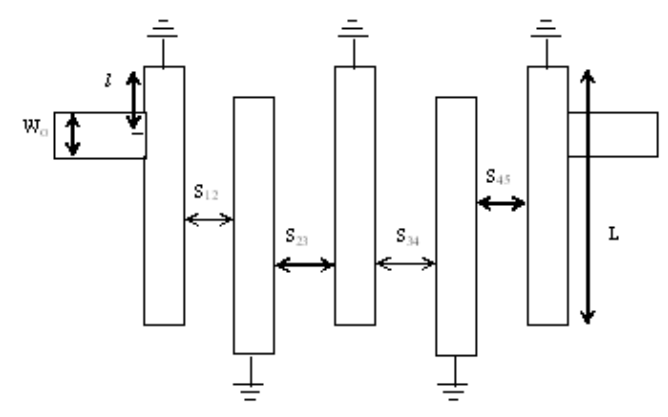

Fig. 1: General structure for interdigital bandpass filter.

In designing the interdigital bandpass filter, the specific frequency, stopband attenuation, attenuation response, and the number of the resonator must be calculated according to the following equations:

$$
\begin{aligned}
& \text { Passband } A=10 \log \left(1+a_{m}^{2}\right) \cos ^{2}\left(n \cos ^{-1} w_{x}^{\prime}\right) \\
& \text { Stopband } A=10 \log \left(1+a_{m}^{2}\right) \cosh ^{2}\left(n \cos ^{-1} w_{x}^{\prime}\right)
\end{aligned}
$$

where, $a_{m}^{2}$ is the ripple height, $n$ is the order of the filter, $w_{x}^{\prime}$ is the bandwidth over which the attenuation has maximum ripple.

The $a_{m}^{2}$ and $w_{x}^{\prime}$ can be calculated from the ripple magnitude by using the eqns. (3) and (4) as follows:

$$
\text { ripple }=10 \log \left(a_{m}^{2}+1\right)
$$




$$
w_{x}^{\prime}=\frac{f_{0}}{f_{2}-f_{1}}\left(\frac{f_{x}}{f_{0}}-\frac{f_{0}}{f_{x}}\right)
$$

where, $f_{0}$ is the centre frequency, $f_{x}$ is the upper frequency, $f_{2}-f_{1}$ is the frequency difference.

From the eqn. 4 , the number of the resonators in the design can be calculated in order to get the accurate pass band of the filter. According to the number of the resonators and the ripple magnitude, the values of $g_{k}$ can be found from the table of reference [12]. From this value, the loaded $Q_{L}$ and the normalized coupling coefficients, $K$ between two resonators can be calculated by using the following equations:

$$
\begin{aligned}
& Q_{L}=f_{0} g_{1} / \nabla f \\
& K_{n, n+1}=\frac{f}{f_{0} \sqrt{\left(g_{n} g_{n+1}\right)}}
\end{aligned}
$$

The length of the resonators can be calculated by using this equation

$$
L=\frac{\lambda_{0}}{4 \sqrt{\varepsilon_{\text {eff }}}}
$$

By taking the value of 0.7 for $W / h$ (width of the substrate over its height) and the substrate dielectric constant, $\varepsilon_{r}$ the effective dielectric constant can be calculated in order to get the length of the resonators.

$$
\varepsilon_{\text {eff }}=\frac{\varepsilon_{r}+1}{2}+\frac{\varepsilon_{r}-1}{2}\left[\left(1+\frac{12 h}{W}\right)^{-1 / 2}+0.04\left(1-\frac{W}{h}\right)^{2}\right]
$$

The last parameter in designing the interdigital bandpass filter is the width of the input and output, $W_{0}$. This parameter can be obtained from impedance $Z_{o}$ from the following eqn. 9.

$$
Z_{0}=\frac{120 \pi}{2 \pi \sqrt{\varepsilon_{\text {eff }}}} \ln \left[\frac{8 h}{W_{0}}+0.25 \frac{W_{0}}{h}\right] ; \quad W_{0} / h<1
$$

The schematic diagram of the interdigital filter is shown in Fig. 2 drawn by using 3D electromagnetic simulator. The center frequency $f_{0}=4 \mathrm{GHz}$, and bandwidth $=0.8 \mathrm{GHz}$ have been chosen for designing the filter. Thefive resonators are assembled on the alumina substrate in the design. The alumina substrate is placed on the ground plane made of copper plate with $0.5 \mathrm{~mm}$ thickness. The filter requires use of grounding of the resonators. The grounding is placed at the side of one end of the resonator and connected to the ground plane via hole through the substrate. The microstrip line is used as the feeding technique. 


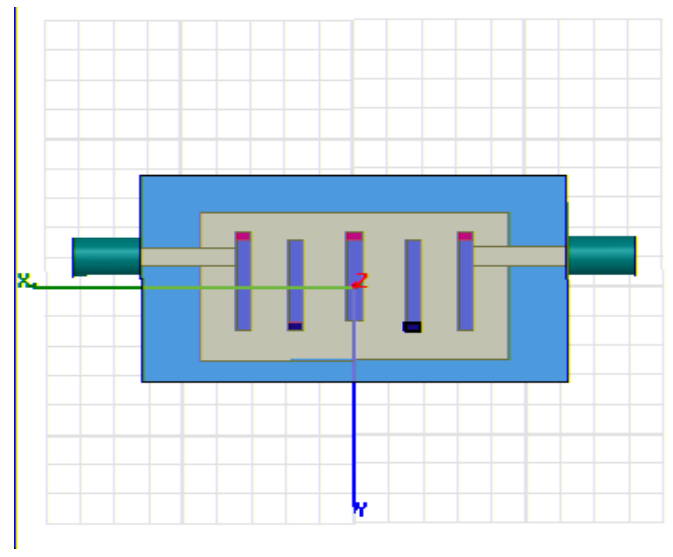

Fig. 2: The layout of inter digital band pass filter.

Figure 3 shows the inter-digital band pass filter response without the MEMS bridge/switches. The frequency response is obtained by using electromagnetic simulator HFSS.

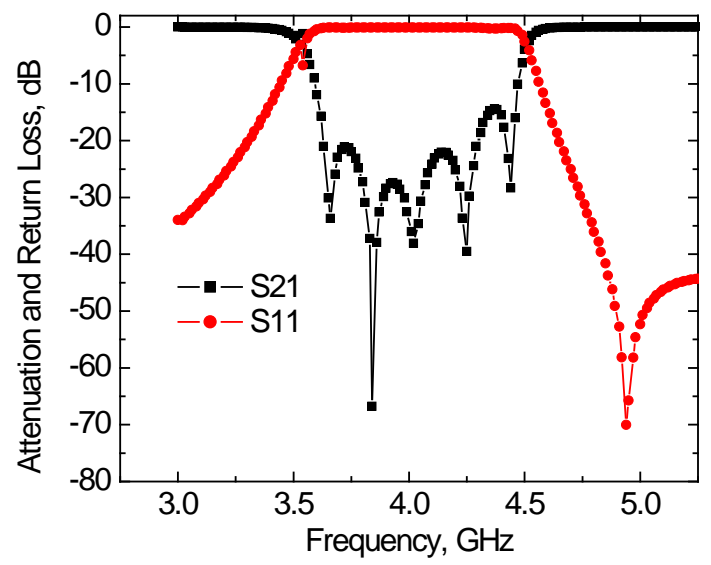

Fig. 3: Simulated results of interdigital filter without MEMS switches.

\subsection{One MEMS Bridge Parallel with the Resonator}

The effect of one MEMS bridge placed above the middle resonator has been studied. The MEMS bridge consists of thin strip of metal (membrane) and insulator that is fixed at both ends. It is suspended above an electrode with spacing, forming a capacitor between these two conductors. The bridge is considered silicon nitride of dielectric constant $\varepsilon_{r}=$ 6.8 with copper coating. The dimension of the bridge is $1.689 \mathrm{~mm} \times 14 \mathrm{~mm} \times 0.1 \mathrm{~mm}$. The structure is shown in Fig. 4. The insertion loss and return loss areshown in Fig. 5. The result shows that the bandwidth is decreasing as the distance between the bridge and the resonator becomes closer. The upper frequency is nearly maintained while the lower frequency is changing. Since air is separating the electrode and the switch, the up-state capacitance is small. By applying a voltage the upper membrane is deflected by the electrostatic force, and the membrane will snaps down to the opposite electrode. The spacing is also reduced and the capacitance is increased. Electrostatic force between the top and bottom electrodes actuates the switch. The variation in capacitance will disturb the 
frequency response of the filter. Furthermore, the fringing fields capacitances of MEMS switches dependson the bridge dimensions and height. As a result the lower cutoff frequency decreases to lower value. The results are summarized in Table 1.

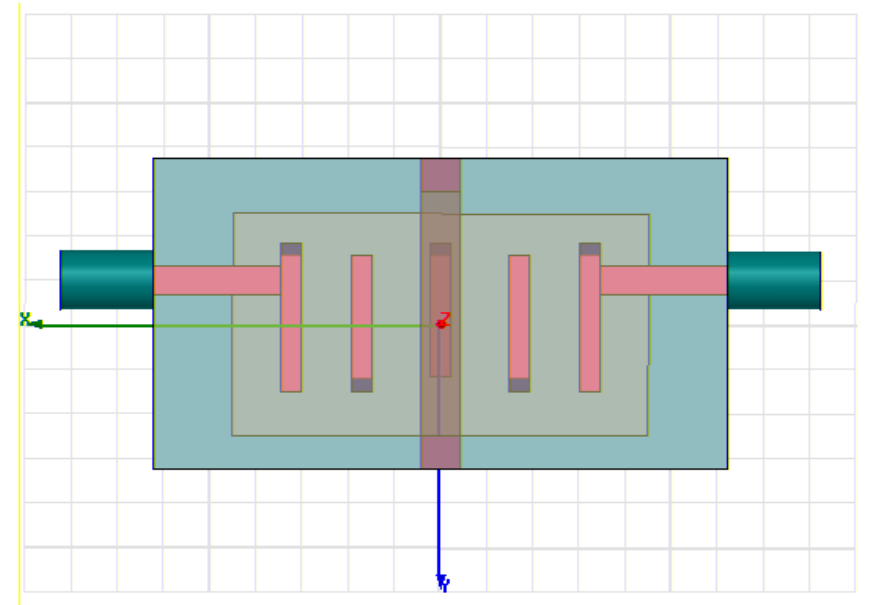

Fig. 4: The layout of the filter with RF MEMS bridge placed above the middle resonator.
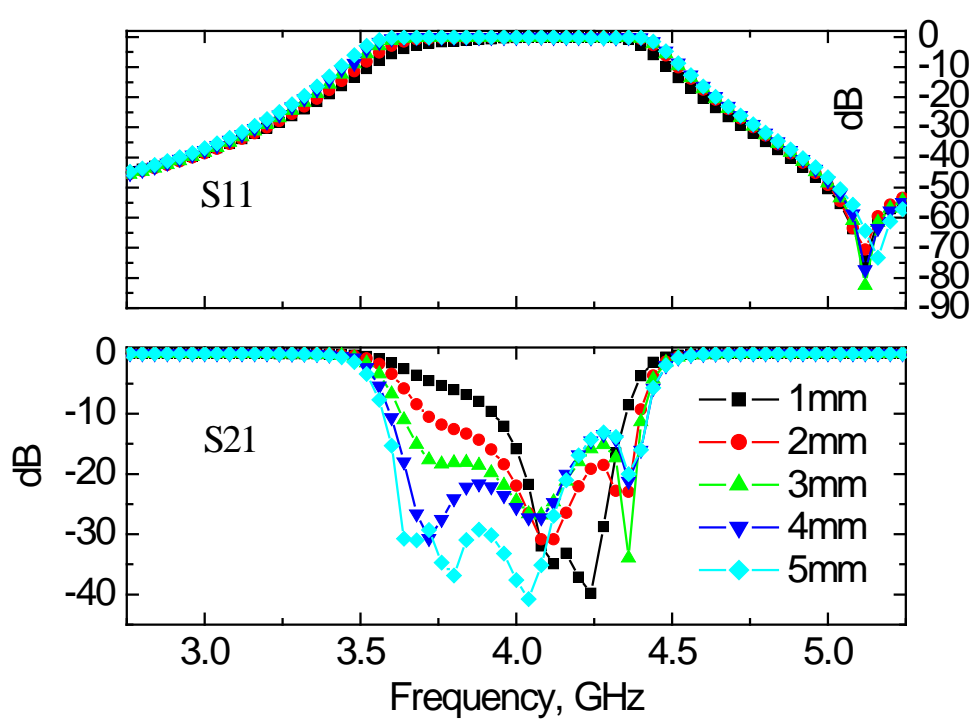

Fig. 5: Simulated results of interdigital filter with single MEMS switch placed parallel to the center resonator.

Table 1: The result for the filter with five MEMS bridges parallel with the resonator.

\begin{tabular}{|c|c|c|c|c|}
\hline $\begin{array}{c}\text { Distance, } \mathbf{d} \\
(\mathbf{m m})\end{array}$ & $\begin{array}{c}\mathbf{f}_{\mathbf{L}} \\
(\mathbf{G H z})\end{array}$ & $\begin{array}{c}\mathbf{f}_{\mathrm{U}} \\
(\mathbf{G H z})\end{array}$ & $\begin{array}{c}\mathbf{f}_{\mathrm{C}} \\
(\mathbf{G H z})\end{array}$ & $\begin{array}{c}\text { Bandwidth } \\
(\mathbf{G H z})\end{array}$ \\
\hline 1 & 3.640 & 4.444 & 4.042 & 0.804 \\
\hline 2 & 3.570 & 4.447 & 4.0085 & 0.877 \\
\hline 3 & 3.544 & 4.455 & 3.9995 & 0.911 \\
\hline
\end{tabular}




\begin{tabular}{|l|l|l|l|l|}
\hline 4 & 3.520 & 4.454 & 3.987 & 0.934 \\
\hline 5 & 3.505 & 4.467 & 3.986 & 0.962 \\
\hline
\end{tabular}

\subsection{Two MEMS Bridge Parallel with the Resonator}

The performance of the filter is analyzed by turning the switch on and off by placing two bridges parallel to the resonator places beside the centre resonator. It is observed from Fig. 6 that there is not much in difference in bandwidth variation between the filter with placing only single MEMS bridge in parallel with the resonator. However, for two bridges, the bandwidth for the down-state is much wider than the single bridge.
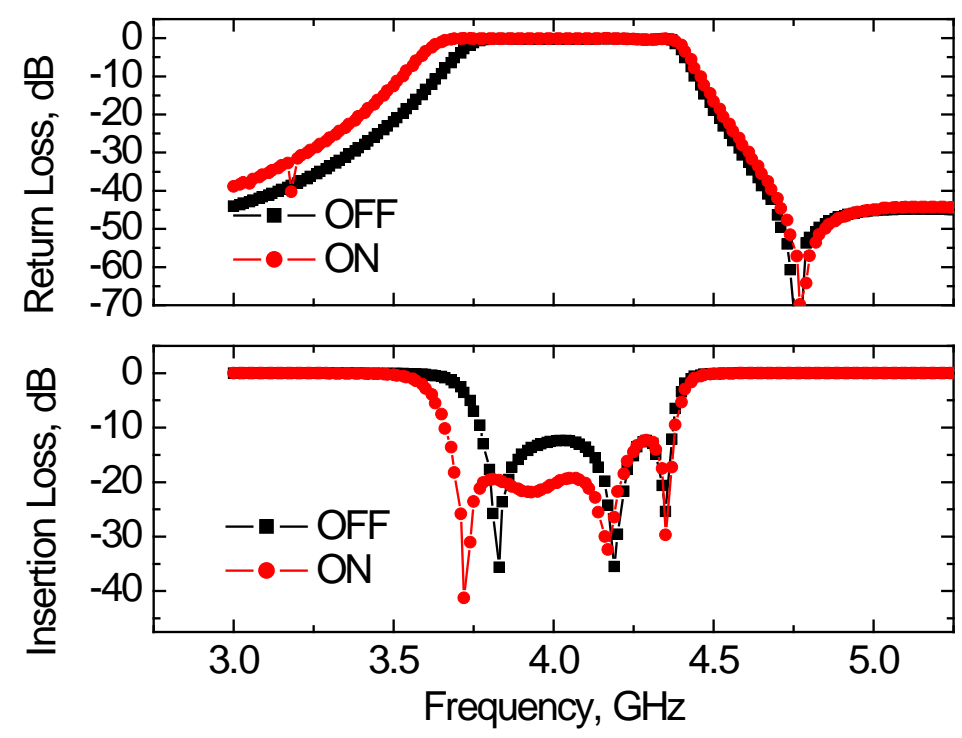

Fig. 6: Simulated results of interdigital filter with two MEMS switches placed parallel beside the center resonator.

\subsection{One MEMS Bridge Perpendicular to the Resonator}

The effect of MEMS bridge placed perpendicularly above the resonator has been studied. The bridge is made of silicon nitride with $0.889 \mathrm{~mm} \times 24.885 \mathrm{~mm} \times 0.1 \mathrm{~mm}$ with copper coating as shown in Fig. 7.

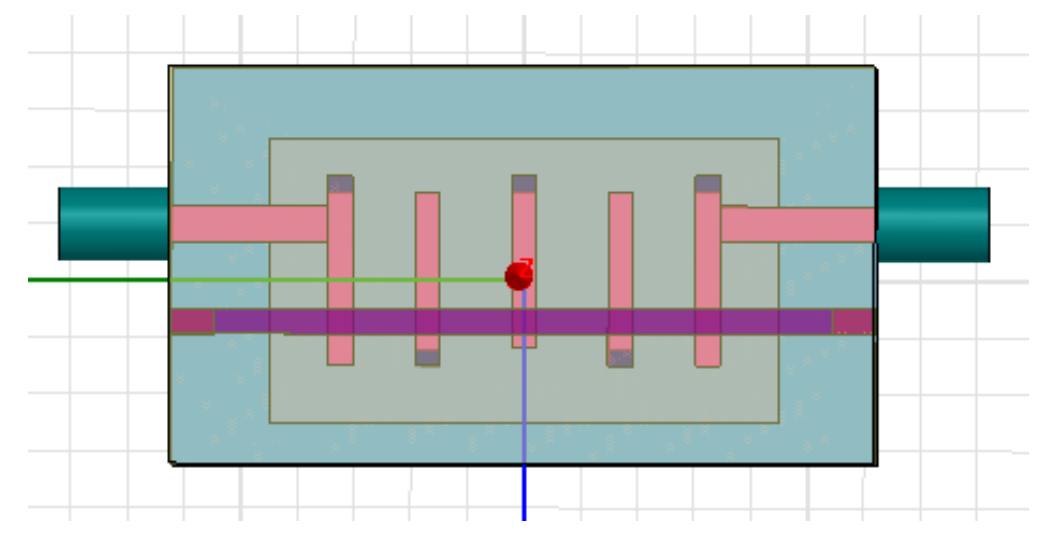


Fig. 7: The layout of the filter with RF MEMS bridge placed perpendicularly above the resonator.

The performance of the filter is analyzed by turning the switch on and off. Figure 8 shows the frequency response of the filter when the MEMS switch is down-state and upstate. From Fig. 8, it can be observed that the bandwidth varies within the frequency range of $3.6 \mathrm{GHz}$ to $4.4 \mathrm{GHz}$. In the off-state position, the bandwidth and center frequency has not been changed. On the other hand, when the MEMS bridge was moved down (on-state position), the gap was reduced and therefore the capacitance was increased and the bandwidth tends to degrade since the capacitive part of the resonators was changed. In this design, the lower frequency remains, while the upper frequency decreases when the switch is in the down-state. It is also observed that the lower frequency shifted to lower frequency region for the case of parallel bridge whereas the higher frequency shifted to high frequency for the case of perpendicular bridge.

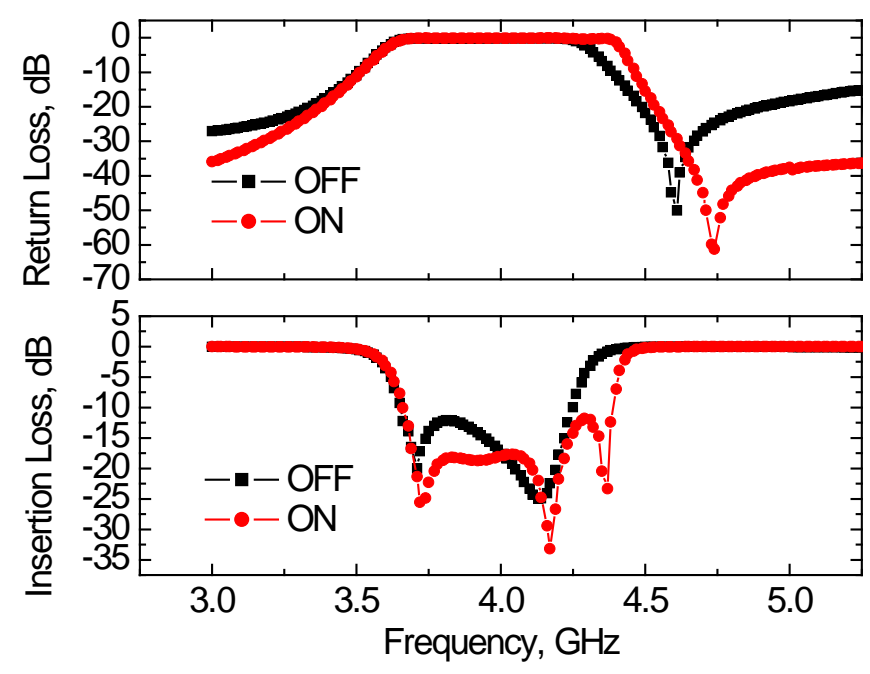

Fig. 8: Simulated results of interdigital filter with MEMS switch placed perpendicular to the center resonator.

\subsection{MEMS Bridges Parallel and Covering All the Resonators}

The filter was designed with MEMS bridges placed on the top of the each resonator, and all the MEMS bridges had the same width as the resonator. The layout of the filter is depicted in Fig. 9. The performance of the filter is analyzed by varying the height of the resonators.

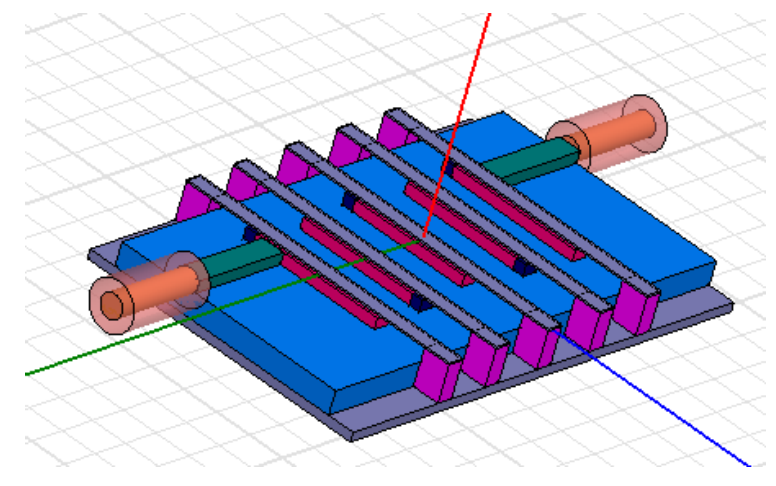


Fig. 9 :The layout of the filter with RF MEMS bridges covered by all the resonators.

The frequency response of the filter is shown in Fig. 10 for different MEMS height. It is observed that the variation in bandwidth is independent of frequencywhen the height of the bridge varies. In this configuration, the bandwidth is dependent on the height of the bridge, as it is reducing the gap of the bridge. The resonators will result in increasing the capacitance of the bridge. Therefore, increasing or decreasing the bridge capacitance enables the bandwidth to be tuned within the same pass band. Table 2 depicted the center frequency and bandwidth for the filter.
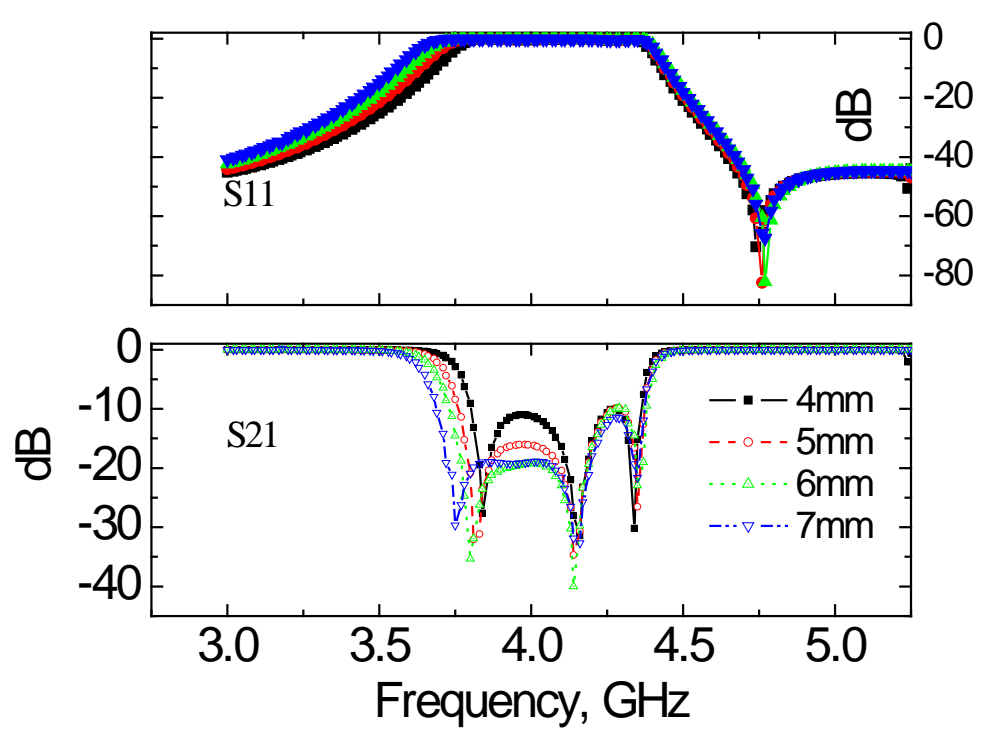

Fig. 10: Simulated results of interdigital filter with MEMS bridges covering all the resonators for various heights.

Table 2: The simulation results for one MEMS bridge placed parallel with the resonator.

\begin{tabular}{|c|r|r|r|r|}
\hline $\begin{array}{c}\text { Height of the } \\
\text { bridge, (mm) }\end{array}$ & $\begin{array}{c}\mathbf{f}_{\mathbf{L}} \\
(\mathbf{G H z})\end{array}$ & $\begin{array}{c}\mathbf{f}_{\mathbf{U}} \\
(\mathbf{G H z})\end{array}$ & $\begin{array}{c}\mathbf{f}_{\mathbf{C}} \\
(\mathbf{G H z})\end{array}$ & $\begin{array}{c}\text { Bandwidth } \\
(\mathbf{G H z})\end{array}$ \\
\hline 4.0 & 3.76 & 4.36 & 4.06 & 0.6 \\
\hline 5.0 & 3.72 & 4.38 & 4.05 & 0.66 \\
\hline 6.0 & 3.68 & 4.4 & 4.04 & 0.72 \\
\hline 7.0 & 3.62 & 4.4 & 4.01 & 0.78 \\
\hline
\end{tabular}

Figure 11(a) shows the equivalent circuit model of 5 -wire interdigital filter. The couplings are restricted to be between adjacent lines, i.e. non-adjacent line couplings are assumed to be zero and also neglecting all the fringing capacitances. The equivalent circuit is going to modify by placing the MEMS bridge on the interdigital filter as shown in Figs. 11(b)-11(d). If the MEMS bridge is placed on the center line the equivalent circuit is 
shown in Fig. 11(b) where $\mathrm{C}_{\text {MEMS }}$ is series connected to the 3rd branch. If the MEMS bridge is placed along all the interdigital filter wire, MEMS capacitances are distributed 

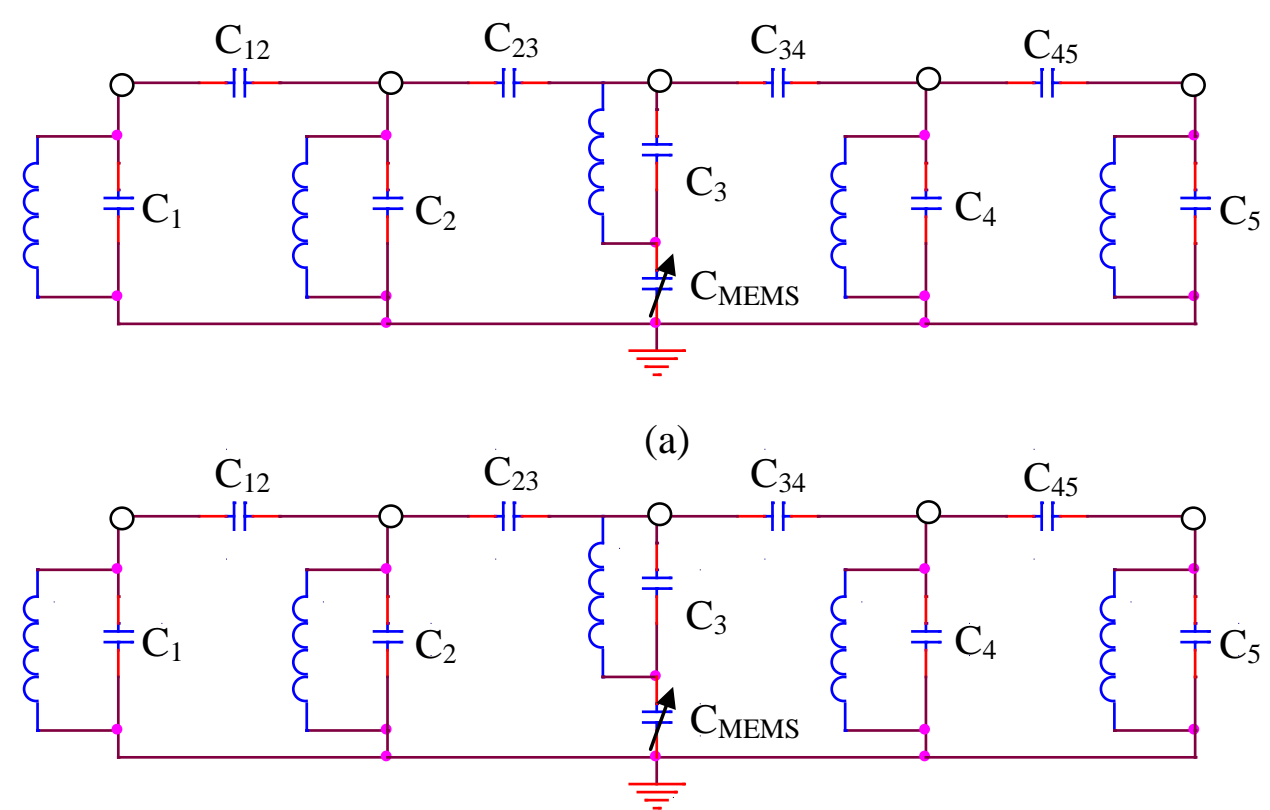

(b)
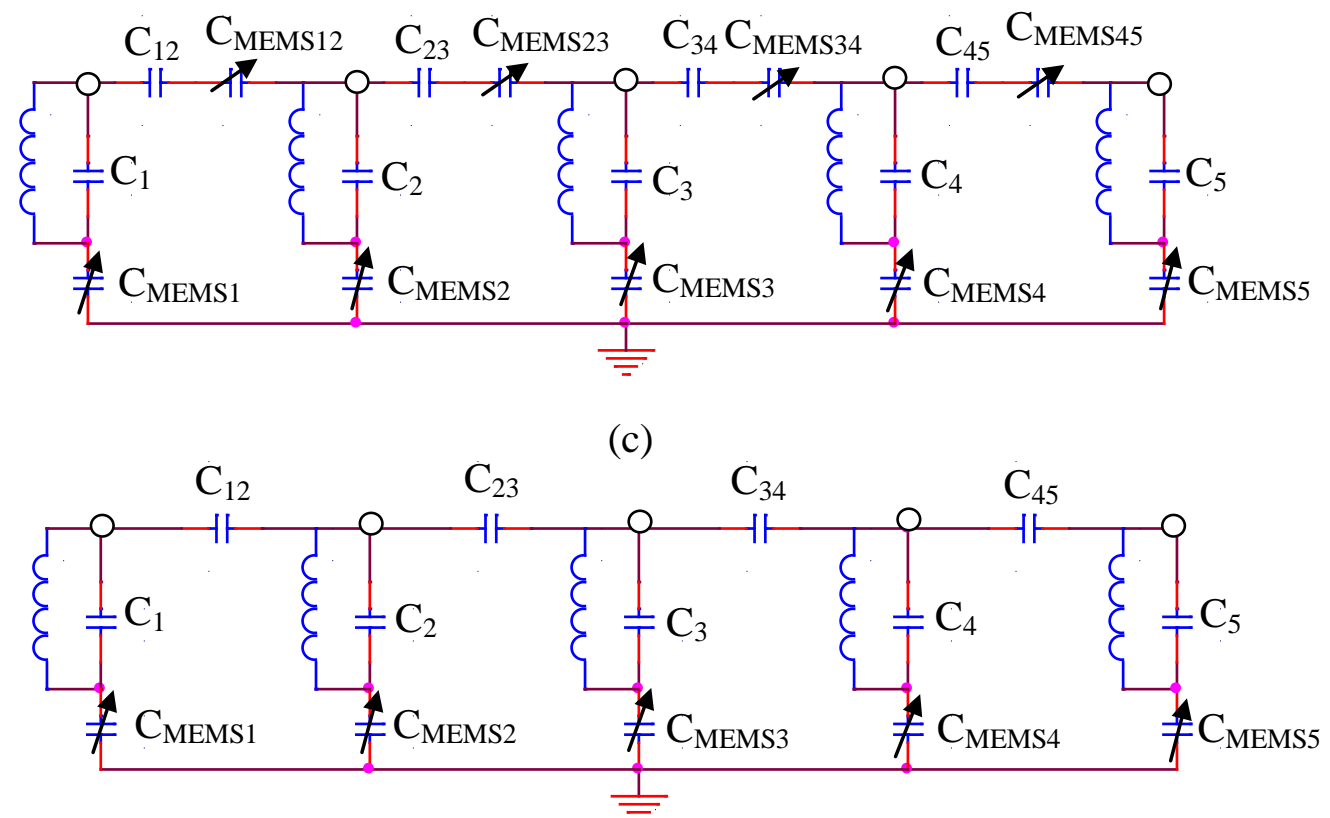

(d)

Fig. 11: Static equivalent capacitance model of proposed 5-wire interdigital filter (a) without MEMS, (b) MEMS bridge placed on centre line, (c) MEMS bridges placed across all the lines, and (d) MEMS bridges placed on each line. 
throughout the coupling capacitance. All the branches are connected to the ground as shown in Fig. 11(c), whereas MEMS capacitances are connected to all the branches without distributed in the coupling capacitance if MEMS bridges are placed parallel to the interdigital line as shown in Fig. 11(d). The nodes connected to $C_{1}$ and $C_{2}$ are input and output nodes respectively. The variation of the MEMS capacitances results the variation of the frequency response of the bandpass filter.

\section{CONCLUSIONS}

The criteria of RF filters with a higher performance, smaller size, lighter weight, lower cost, low loss, and high selectivity can be achieved by using RF MEMS technology. The designing of the interdigital band pass filter has been presented with the analysis of the frequency responses of the filter. The use of capacitive RF MEMS switch in the application of interdigital band pass filter is investigated in this paper. The designs indicate that the application of capacitive RF MEMS switches in the tunable filter enables the filter to tune the bandwidth in the frequency range of $3.6 \mathrm{GHz}$ to $4.4 \mathrm{GHz}$, which was the range for the filter design without MEMS switch. The tunability has made the filter suitable to be used in different types of application.

\section{ACKNOWLEDGEMENT}

The authors thank the Ministry of Higher Education- Fundamental Research Grant Scheme, FRGS Grant Ref: IIUM/504/RES/G/14/3/05/FRGS for funding this research through the International Islamic University Malaysia (IIUM).

\section{REFERENCES}

[1] I.C. Hunter, L. Billonet, B. Jarry, and P. Guillon, "Microwave Filters-Applications and Technology”. IEEE Trans. Microwave Theory \& Tech. 50(3), pp.794-804, 2002.

[2] J. Uher, and J. R. Hofer, “Tunable microwave and milimeter-wave band pass filters". IEEE Trans.Microwave Theory Tech., 39(4), pp. 643-653, 1991.

[3] G. L. Matthaei, E. Young, and E. Jones, "Microwave Filters, Impedance- Matching Networks, and Coupling Structures”. Norwood, MA: Artech House, 1980

[4] G. M. Rebeiz, "RF MEMS Theory, Design, and Technology”. John Wiely and Sons, 2003.

[5] K. Entesari, and G. M. Rebeiz, “A 12-18 GHz 3 Pole RF MEMS Tunable Filter”. IEEE Trans. on Microwave Theory Tech., 53 (8), pp. 2566-2571, 2005.

[6] A. Pothier, J. C. Orlianges, and E. Zheng "Low Loss 2-Bit Bandpass Filters Using MEMS DC contact switches”. IEEE Trans. on Microwave Theory Tech. 53, pp. 354-360, 2005.

[7] M. Miao, J. Bu, and L. Zhao, "A Bulk Micromachined Tunable Microwave Lowpass Filter for 10-15GHz Wireless/Satellite Communication”. Proceedings of the 3rd IEEE Int. Conf. on Nano/Micro Engineered and Molecular System, China, pp. 524- 528, 2008. 
[8] C. Rauscher, "Reconfigurable band pass filter with a three-to-one switchable passband width”. IEEE Trans. Microw. Theory Tech., vol. 52, no. 3, pp. 573-577, 2003.

[9] W.H. Tu, and K. Chang, "Piezoelectric Transducer-Controlled Dual-Mode Switchable Bandpass Filter”. IEEE Microw. And Wireless Components Lett, vol. 17, no. 3, pp. 199201, 2007.

[10] K. Entesari, K. Obeidat, A.R. Brown, and G. Rebeiz, "A 25-75-MHz RF MEMS Tunable Filter”. IEEE Trans. Microw. Theory Tech., vol. 55, no. 11, pp. 2399-2405, 2007.

[11] J. S Wong and M.J. Lanchester, “Microstrip Filters for RF/Microwave Applications”. John Wiley \& Sons Inc, 2001.

[12] A. Das and S. D. Das, "Microwave Engineering”. McGraw Hill International Edition, 2001. 